Check for updates

Cite this: RSC Adv., 2019, 9, 22567

Received 14th May 2019

Accepted 4th July 2019

DOI: $10.1039 / c 9 r a 03633 d$

rsc.li/rsc-advances

\section{The characteristics of high-quality Yb:YAG single crystal fibers grown by a LHPG method and the effects of their discoloration}

\author{
Tao Wang, ${ }^{a}$ Jian Zhang, ${ }^{\text {ab }}$ Na Zhang, (D) a Siyuan Wang, ${ }^{a}$ Baiyi Wu, ${ }^{a}$ Zhitai Jia (D) *ab \\ and Xutang Tao (D) *ab
}

Single crystal fibers (SCFs), especially ytterbium (Yb) doped crystal fibers, have great potential in the field of high-power lasers. Colorless Yb:YAG single crystal fibers were fabricated using a laser heated pedestal growth (LHPG) method with a diameter fluctuation of less than $2 \%$ and a length to diameter ratio greater than 320 : 1. An abnormal color issue exists with respect to Yb:YAG crystals. The origin of coloration was studied via density functional theory, single-crystal X-ray diffraction, XPS and Raman spectroscopy and it was confirmed that the cyan coloration of Yb:YAG crystals is due to oxygen vacancies. Yb:YAG SCFs prepared via the LHPG method could avoid this type of defect due to the large specific surface area and melt convection caused by surface tension. The fundamental properties of the cyan Yb:YAG crystal source rod and colorless Yb:YAG SCFs were systematically investigated. The colorless Yb:YAG SCFs have higher infrared transmittance and thermal conductivity. The distributions of $\mathrm{Yb}^{3+}$ along the radial and axial directions were also measured. Meanwhile we demonstrated the propagation loss and a fiber laser using the colorless Yb:YAG SCFs, obtaining a minimum loss coefficient of $0.008 \mathrm{~dB} \mathrm{~cm}^{-1}$ and a maximum continuous-wave (CW) output power of $3.62 \mathrm{~W}$. The colorless Yb:YAG SCFs with good thermal conductivity, low propagation loss, wide transparency and uniform ion distribution show promise for acting as the host material in single-mode lasers.

\section{Introduction}

Nowadays, with the explosive development of solid-state lasers, high-power lasers are gradually being applied to materials processing, information and communication technology, medical treatment and many other fields. Therefore, increasing the output power and reducing thermal effects present huge challenges for traditional solid-state gain media, such as glass, ceramics and crystals. $^{1-8}$ Because of their excellent optical conversion efficiency, good beam quality and lower maintenance cost, fiber lasers are gradually becoming a new hotspot in the field of solid-state lasers. Their output power has reached 10 $\mathrm{kW}$ in continuous-wave form, mainly in ship-borne lasers and laser weapons. Compared with traditional solid-state laser media, fibers have a larger specific surface area, which greatly improves the thermal management of the laser. However, due to limitations inherent in the material properties, it is difficult to obtain higher output powers with conventional glass fibers. Therefore, crystal fibers with higher thermal conductivity, better mechanical properties and higher damage thresholds

${ }^{a}$ State Key Laboratory of Crystal Materials, Shandong University, Jinan, 250100, China.E-mail: z.jia@sdu.edu.cn; txt@sdu.edu.cn

${ }^{b}$ Key Laboratory of Functional Crystal Materials and Device, Ministry of Education, Jinan 250100, China can be used to promote the development of next-generation high-power lasers. ${ }^{9-17}$

Yttrium aluminium garnet (YAG) belongs to a cubic system, with the unit cell parameter $a=12.0089 \AA$. YAG crystals have good physical and chemical stabilities and have been widely used in solid-state lasers..$^{18}$ At the same time, YAG crystals can achieve high doping concentrations because of the similar sizes of yttrium atoms and rare-earth atoms. Compared to traditional silica fibers, YAG crystal fibers have a lower Brillouin gain coefficient, a higher laser damage threshold and higher thermal conductivity. The theoretical single-mode output of a YAG SCF laser is more than 100 times that of a glass fiber laser. ${ }^{19-23}$

Yb-doped YAG crystals are an excellent laser medium. $\mathrm{Yb}^{3+}$ ions have a simple electronic structure, with only one excited state $\left(2 \mathrm{~F}_{5 / 2}\right)$ above the ground state $\left(2 \mathrm{~F}_{7 / 2}\right)$, avoiding thermal effects due to up-conversion and excited state absorption. Moreover, the theoretical segregation coefficient of $\mathrm{Yb}^{3+}$ is close to 1 , which is beneficial for miniaturizing devices. Compared to $\mathrm{Nd}^{3+}, \mathrm{Yb}^{3+}$ is well suited for IR InGaAs diode laser pumping between 900 and $980 \mathrm{~nm}$. Meanwhile, the close absorption (900-980 nm) and emission wavelengths (980-1100 nm) lead to a low thermal load $\left(11 \%\right.$ relative to $30 \%$ in $\mathrm{Nd}^{3+}$-doped laser hosts), thereby significantly improving the slope efficiency, which reaches almost $90 \%$ theoretically. ${ }^{24-26}$ Therefore, Yb:YAG crystals are considered to be the most promising fiber material 
for $1 \mu \mathrm{m}$ band lasers. ${ }^{27-30} \mathrm{~A}$ continuous-wave output power of $251 \mathrm{~W}$ obtained by a Yb:YAG SCF laser is also reported to be a record in the field of crystal fiber lasers. ${ }^{31}$

However, it is interesting to find that commercial Yb:YAG crystals are usually different shades of cyan, and the color always deepens with an increase in the crystal size. This color issue relating to Yb:YAG crystals should be the primary problem to be solved, especially if they are going to be used in highpower lasers. Usually, most commercial Yb:YAG crystals are grown via the Czochralski method. An inert or reductive atmosphere may be the cause of the discoloration. ${ }^{32,33}$ This color issue has puzzled people for a long time, but the origin of the coloration of Yb:YAG crystals has never been systematically justified.

In this work, we have successfully fabricated colorless and transparent Yb:YAG SCFs using Yb:YAG bulk crystals with a cyan color in both oxygen-rich and inert atmospheres via a LHPG method. Besides, we have designed an experimental scheme to seek the origins of the coloration. Some optical and thermal properties of cyan Yb:YAG crystal source rods and colorless YAG SCFs were also compared. The second part of this paper relates to the fundamental characteristics of Yb:YAG SCFs, including diameter fluctuations, crystal quality, propagation loss and ion distribution. A Yb:YAG fiber laser is also reported in this paper.

\section{Experimental}

\subsection{Growth of single crystal fibers}

A schematic diagram detailing the LHPG method is shown in Fig. 1. Generally, solid-phase sintered ceramic or bulk crystals are used as the source rods and seed crystals. We used a $2 \% \mathrm{Yb}$ doped YAG bulk crystal as the source rod; moreover, an undoped YAG crystal orientated in the $\langle 111\rangle$ direction was selected as the seed crystal. The ring $\mathrm{CO}_{2}$ laser is focused on the top of the source rod to form a melting zone. The seed crystal is immersed in the melting zone and kept at the center of the source rod to stable the melting zone. Approximately 50 to $60 \mathrm{~W}$ of laser

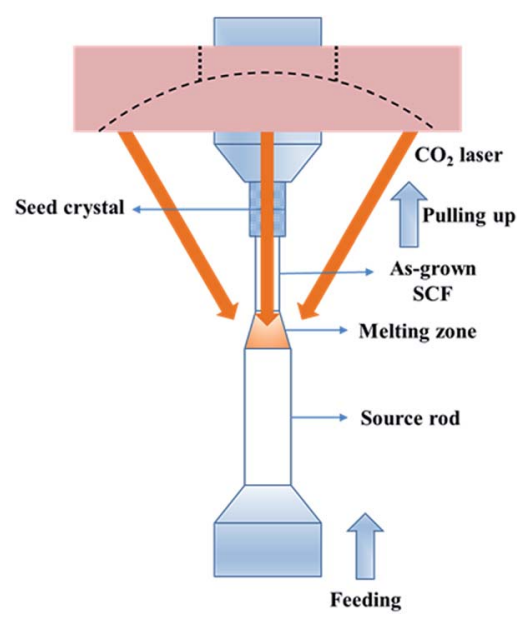

Fig. 1 A schematic diagram of the LHPG technique. power is needed to melt a $3 \mathrm{~mm}$-diameter source rod. The growth rate is about $0.2-1 \mathrm{~mm} \mathrm{~min}^{-1}$ which means one hundred millimeters of fiber can be obtained in $2 \mathrm{~h}$. The ratio of the pulling speed to the feeding speed determines the diameter of the crystal fiber; generally, this is no more than $9: 1$, converting to a diameter ratio of $3: 1$. Therefore, some SCFs need to be grown twice to obtain finer examples. Compared with other crystal growth methods, there is no crucible in the LHPG method, which greatly improves the purity of the raw materials. At the same time, non-crucible growth conditions enrich the growing atmosphere. We can use not only inert atmospheres but also air or even oxygen atmospheres during crystal growth.

As shown in Fig. 2, the Yb:YAG crystal source rods are cyan, however, after processing via the LHPG method, colorless and transparent crystal fibers were obtained without any postprocessing. In this paper, we obtained $\Phi 2.0 \times 50 \mathrm{~mm}^{3}$ Yb:YAG SCFs with a pulling ratio of $3: 1$; these were then grown twice, obtaining $\Phi 0.8 \times 260 \mathrm{~mm}^{3}$ and $\Phi 0.6 \times 150 \mathrm{~mm}^{3}$ Yb:YAG SCFs with pulling ratios of $5: 1$ and $9: 1$, and a length-todiameter ratio greater than $320: 1$.

\subsection{Single-crystal X-ray diffraction}

In order to compare the cell parameters of the cyan source rod and the colorless single crystal fiber, single-crystal X-ray diffraction studies were performed using D8 VENTURE apparatus and the patterns were obtained using a Bruker SMART APEX-III diffractometer equipped with a CCD area detector and using graphite-monochromated Mo-K $\alpha$ radiation $(\lambda=0.71073$ $\AA$ ) at $296 \mathrm{~K}$. Diffraction data collection and unit-cell refinement were performed using the INTE-GRATE program with APEX-3 software.

\subsection{X-ray photoelectron spectroscopy}

XPS measurements were carried out to verify the existence of $\mathrm{O}^{2-}$ ions using Thermo ESCALAB 250XI apparatus. The tested samples were wafers of the cyan source rod and as-grown colorless SCFs.

\subsection{Optical properties}

The UV-visible extinction spectra of the Yb:YAG crystal source rod and as-grown Yb:YAG SCFs were recorded over a wavelength range extending from 200 to $900 \mathrm{~nm}$ using a Cary 50 spectrophotometer.

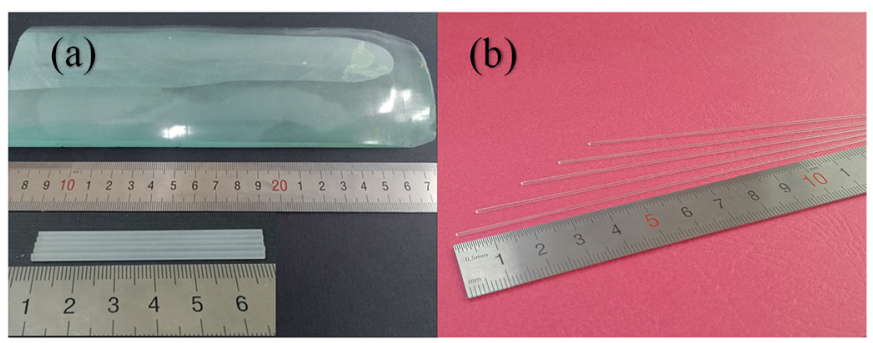

Fig. 2 (a) The Yb:YAG bulk crystal and processed source rods. (b) Asgrown Yb:YAG SCFs. 
The RT optical transmission spectra of the Yb:YAG crystal source rod and as-grown Yb:YAG SCFs were obtained using a PE IR SPECTRUM ASCII PEDS 1.60 Fourier transform infrared (FTIR) spectrometer over the wavenumber range of 450$7800 \mathrm{~cm}^{-1}$.

The RT Raman spectra of the Yb:YAG crystal source rod and as-grown Yb:YAG SCFs were recorded using a Horiba Jobin Yvon LabRAM HR Raman spectrometer equipped with a liquid $\mathrm{N}_{2}$ cooled Ge detector. A solid laser power of $150 \mathrm{~mW}$ at $532 \mathrm{~nm}$ was used for excitation.

\subsection{Thermal properties}

The specific heats of the Yb:YAG crystal source rod and asgrown Yb:YAG SCFs were measured using a differential scanning calorimeter (DSC-Q20) from 30 to $295{ }^{\circ} \mathrm{C}$ at a heating rate of $5{ }^{\circ} \mathrm{C} \min ^{-1}$.

The thermal conductivities were measured using the Thermal Transport Option (P670B) of a Quantum Design Physical Property Measurement System (PPMS) from 4-400 K with a liquid nitrogen cooling system. The cooling and heating rates selected in the experiment were $6{ }^{\circ} \mathrm{C} \min ^{-1}$ and $0.5{ }^{\circ} \mathrm{C} \min ^{-1}$, respectively.

\subsection{Computational details}

Geometry optimization calculations were carried out on Yb:YAG based on density functional theory (DFT) with the PerdewBurke-Ernzerhof (PBE) generalized gradient approximation (GGA) functional for the exchange correlation function, implemented using the Vienna $\mathrm{Ab}$ initio Simulation Package (VASP). ${ }^{34-36}$ The energy cutoff was set to $450 \mathrm{eV}$, and the structure relaxation convergence threshold was set to $10^{-5} \mathrm{eV}$ in energy terms and $0.02 \mathrm{eV}^{-1}$ in force terms. A 2 in2 in2 Monkhorst-Pack mesh for Brillouin zone integration was used during geometry calculations. The structure of Yb:YAG is shown in Fig. 3. In order to explore whether the existence of $\mathrm{Yb}^{3+}$ will change the oxygen vacancy formation trend, we compared the formation energies of oxygen vacancies at different $\mathrm{Yb}^{3+}$ doping concentrations. The absorption spectra and refractive indices under different defect conditions were also simulated.

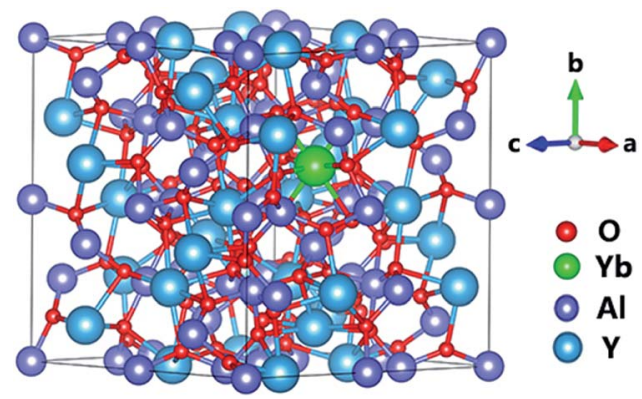

Fig. 3 The optimized Yb:YAG crystal structure. The red, green, purple, and blue spheres represent $\mathrm{O}, \mathrm{Yb}, \mathrm{Al}$, and $\mathrm{Y}$ atoms, respectively.

\subsection{Diameter fluctuation}

Diameter fluctuation is one of the most important parameters for evaluating the quality of single crystal fibers, as it reflects the stability of the crystal growth process. In this paper, the diameter fluctuations of Yb:YAG SCFs were characterized using an LS-9000 laser micrometer. SCFs with diameters of $0.6 \mathrm{~mm}$ and $0.8 \mathrm{~mm}$ were selected as the samples. In order to improve the accuracy of the experiments, we took measurements every millimeter and repeated measurements 100 times for each sample.

\subsection{High-resolution X-ray diffraction (HRXRD)}

HRXRD was performed using a Bruker D8-discover diffractometer equipped with a four-crystal $\mathrm{Ge}(220)$ monochromator set for $\mathrm{Cu}-\mathrm{K} \alpha$ radiation $(\lambda=1.54056 \AA)$. The accelerating voltage and tube current of the generator were $40 \mathrm{kV}$ and $20 \mathrm{~mA}$, respectively. The step time and step size were set to $0.5 \mathrm{~s}$ and $0.001^{\circ}$, respectively. A double-sided, polished, (111)-oriented Yb:YAG SCF wafer was used as the HRXRD sample with dimensions of $\Phi 0.8 \times 1.0 \mathrm{~mm}^{3}$.

\subsection{Laue back-reflection measurements}

To further determine the crystallinity of the SCFs, Laue backreflection measurements were carried out using a real-time back-reflection Laue camera system (Multiwire MWL 120 with Northstar software) to further assess the crystalline perfection of the Yb:YAG SCFs. A (111)-oriented YAG SCF with dimensions of $\Phi 0.6 \times 50 \mathrm{~mm}^{3}$ was employed for the Laue diffraction measurements.

\subsection{Electron probe microanalysis (EPMA)}

The distributions of $\mathrm{Yb}^{3+}$ along the radial and axial directions were characterized using EPMA-1720H apparatus (Shimadzu, Japan). The accelerating voltage and tube current of the generator were $15 \mathrm{kV}$ and $100 \mathrm{nA}$, respectively. The distributions were measured along three diameter directions and the radial direction. 300 points were selected for each set of experiments and they were measured for $0.5 \mathrm{~s}$ per point. A double-sided polished Yb:YAG SCF was used as the EPMA sample with dimensions of $\Phi 0.6 \times 5.0 \mathrm{~mm}^{3}$.

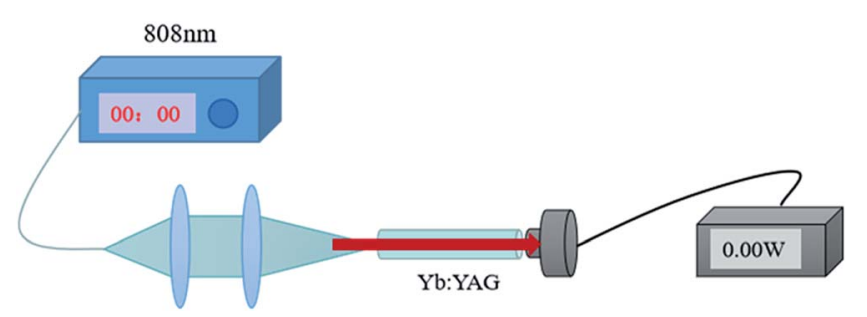

Fig. 4 A simplified schematic diagram of the propagation loss test setup. 


\subsection{Propagation loss}

The propagation loss of a Yb:YAG SCF was measured via a cutback method. A schematic diagram of the propagation loss experiment set-up is shown in Fig. 4. The light source used in the test is a fiber-coupled $808 \mathrm{~nm}$ semiconductor laser, which can effectively avoid loss caused by spectral absorption, improving the accuracy of the test. In order to make the test more accurate, different lengths of fibers were selected for testing.

\subsection{Laser experiments}

The emission spectra of as-grown Yb:YAG SCFs were obtained employing an FLS-980 spectrometer (Edinburgh Instruments).

The experimental apparatus used for the laser experiments is depicted in Fig. 5. A Yb:YAG SCF was pumped using a fibercoupled $940 \mathrm{~nm}$ semiconductor laser with a $200 \mu \mathrm{m}$ core diameter and a NA of 0.22 . The magnification ratio of the focusing system is $1: 1$. The pump beam was focused to a 200 $\mu \mathrm{m}$ diameter, with the focal point located on the input side of the SCF. The input mirror (IM) is a concave mirror, which was coated for high transmission ( $T>98 \%)$ at the pump wavelength (940-976 $\mathrm{nm})$ and high reflectivity $(R>99 \%)$ from 1020 to $1060 \mathrm{~nm}$. The output mirror (OM) was plated with 1020$1070 \mathrm{~nm}$ partially reflective films with transmittances of $3 \%$, $10 \%$ and $30 \%$, respectively. The IM was placed as close as possible to the input side of the SCF, so as to provide as much feedback as possible at the lasing wavelength. The resonant cavity consisted of a flat-concave short cavity structure with a length of $11 \mathrm{~mm}$. In order to reduce the thermal effects of the crystal, the SCF was placed in a copper block and the temperature was set to $15{ }^{\circ} \mathrm{C}$. Spectral detection was performed using an AQ6370D spectrum analyzer and the scanning interval was set to $1020 \mathrm{~nm}$ to $1080 \mathrm{~nm}$ to facilitate observations. $\Phi 0.6 \times 10$ $\mathrm{mm}^{3}$ Yb:YAG SCFs (2 at\% doping) were applied in the laser experiments.

\section{Results and discussion}

\subsection{Unit-cell parameters}

The phenomenon of discoloration occurring in oxide crystals is generally considered to be caused by oxygen defects. In order to determine the type of oxygen deficiency, we collected the unit cell parameters of the cyan source rod and colorless SCFs grown under an inert atmosphere. As shown in Table 1, the cell parameters $(a=b=c=12.03 \AA)$ and cell volume $\left(1743 \AA^{3}\right)$ of the

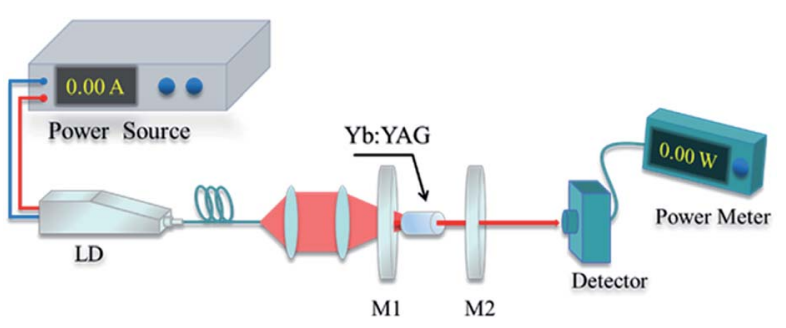

Fig. 5 A schematic diagram of the Yb:YAG SCF laser setup.
Table 1 The unit cell parameters of the source rod and as-grown SCFs

\begin{tabular}{lcccc}
\hline & $a(\AA)$ & $b(\AA)$ & $c(\AA)$ & $V\left(\AA^{3}\right)$ \\
\hline Yb:YAG source rod & 12.03 & 12.03 & 12.03 & 1743 \\
As-grown Yb:YAG SCFs & 12.05 & 12.05 & 12.05 & 1749
\end{tabular}

source rod are smaller than those of the colorless SCFs $(a=b=$ $c=12.05 \AA, V=1749 \AA^{3}$ ). We can speculate from these results that the discoloration of the source rod may be caused by vacancies.

\subsection{XPS studies}

Fig. 6 shows O1s XPS spectra from the Yb:YAG crystal source rod and as-grown Yb:YAG SCFs under an inert atmosphere. The spectra are decomposed into peaks using the Gaussian model. There are three Gaussian peaks at $530.23 \mathrm{eV}, 531.41 \mathrm{eV}$ and $532.40 \mathrm{eV}$. Peak 1 (at $530.23 \mathrm{eV}$ ) represents the lattice oxygen ion content. Peak 2 (at $531.41 \mathrm{eV}$ ) is generally considered to be caused by oxygen vacancies. Peak 3 (at $532.40 \mathrm{eV}$ ) is caused by adsorbed water and adsorbed oxygen. ${ }^{37}$ It can be seen from the figure that the content of oxygen vacancies (peak 2) in the colorless SCFs is lower than that in the source rod, further confirming that the color is related to oxygen vacancies. The large specific surface area of the SCFs and the melt convection caused by surface tension make it possible to avoid the generation of oxygen vacancies, even in an inert environment.

\subsection{Optical properties}

UV-visible extinction spectra of the Yb:YAG crystal source rod and the as-grown Yb:YAG SCFs under an inert atmosphere are shown in Fig. 7(a). We can see that the $\mathrm{Yb}^{3+}$ characteristic peaks of the two samples are consistent, and the absorption coefficients are basically the same. However, the source rod has absorption peaks at $380 \mathrm{~nm}$ and $650 \mathrm{~nm}$. Optical transmission spectra of the source rod and as-grown Yb:YAG SCFs under an inert atmosphere were recorded over the wavelength range of 1300-8000 nm, as shown in Fig. 7(b). We found that the colorless Yb:YAG SCFs exhibit higher infrared transmittance.

Fig. 8 shows simulated absorption spectra and refractive indices under different defect conditions. From the simulation results in Fig. 8(a), we can find that YAG crystals with oxygen vacancies show obvious absorption in the region of 300-
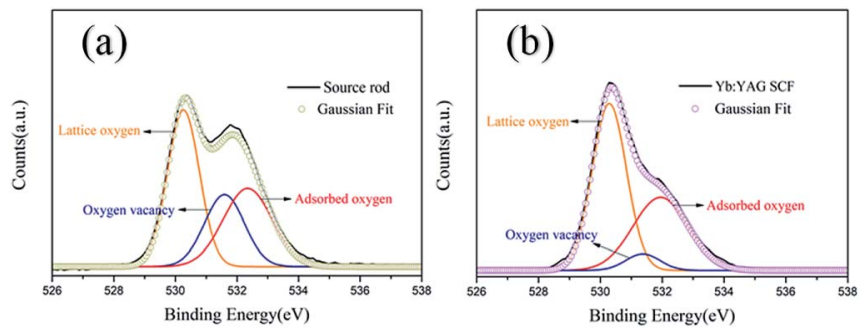

Fig. 6 O1s XPS spectra of (a) the source rod, and (b) as-grown Yb:YAG SCFs. 

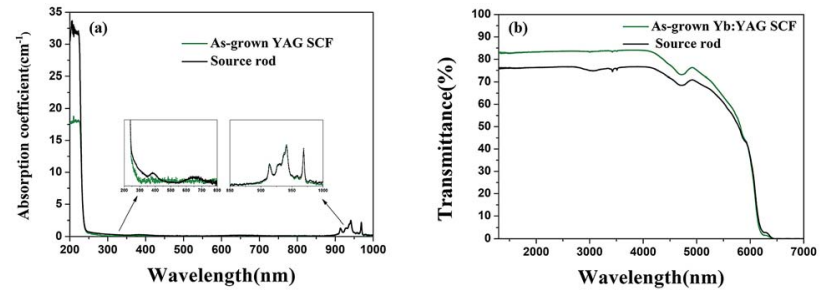

Fig. 7 (a) UV-visible extinction spectra and (b) infrared transmission spectra.
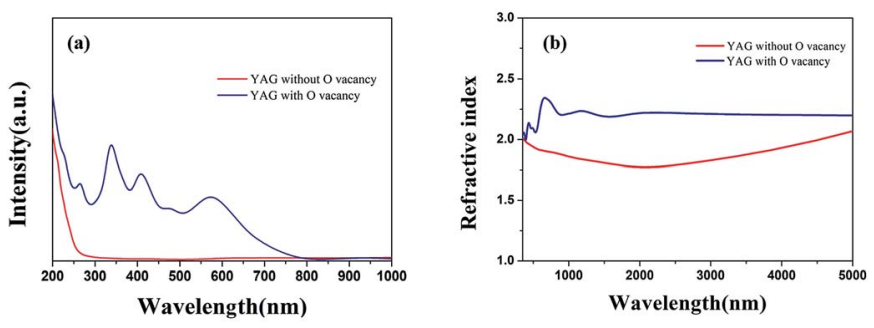

Fig. 8 Calculated (a) absorption spectra and (b) refractive index values under different defect conditions.

$650 \mathrm{~nm}$, which is consistent with the measured absorption spectrum of the discolored Yb:YAG crystal. Fig. 8(b) shows that the Yb:YAG crystal is predicted to possess a higher refractive index in the presence of oxygen vacancies. The Fresnel reflection is positively correlated with differences in the refractive indices at the interface. Therefore, a higher refractive index caused by oxygen vacancies in the Yb:YAG crystal will increase the reflectance at the interface, resulting in greater optical loss. In the measured infrared transmission spectra shown in Fig. 7(b), the transmittance of the discolored sample is lower compared with a colorless sample of the same thickness, which is consistent with the simulation results.

Raman spectra of the source rod and the as-grown SCFs under an inert atmosphere are shown in Fig. 9. By comparison, we found that the intensity of the Raman peak at $546 \mathrm{~cm}^{-1}$ has decreased for the cyan source rod sample and the Raman peak at $1100 \mathrm{~cm}^{-1}$ has disappeared. These two peaks are considered

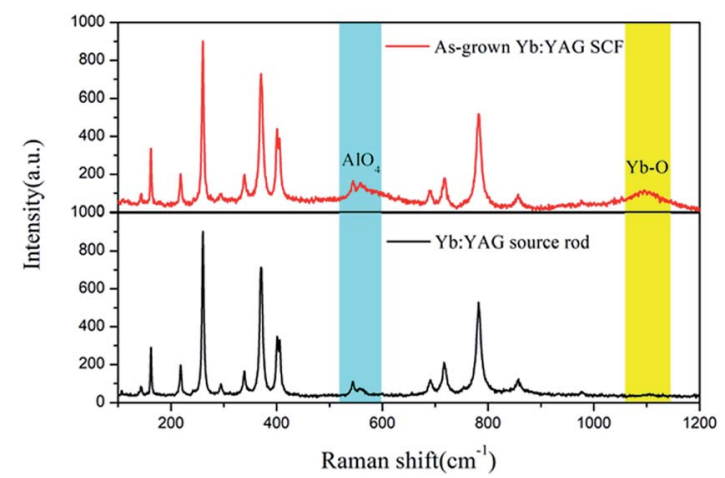

Fig. 9 Raman scattering spectra of the source rod and as-grown Yb:YAG SCFs. to be attributed to the $U_{2}$ mode of $\mathrm{AlO}_{4}$ units and $\mathrm{Yb}-\mathrm{O}$ vibrations, respectively. ${ }^{38}$ These data indicate that the formation of oxygen vacancies in Yb:YAG is related to $\mathrm{Yb}^{3+}$.

\subsection{Thermal properties}

Thermal properties are important for laser crystals, of which thermal conductivity is critical for achieving high energy laser applications.

The specific heat $\left(C_{\mathrm{v}}\right)$ values of the Yb:YAG crystal source rod and the colorless Yb:YAG SCFs grown under an inert atmosphere as a function of temperature are shown in Fig. 10. As can be seen, the specific heat of the Yb:YAG SCF increases from $0.679 \mathrm{~J} \mathrm{~g}^{-1} \mathrm{~K}^{-1}$ at $30{ }^{\circ} \mathrm{C}$ to $0.973 \mathrm{~J} \mathrm{~g}^{-1} \mathrm{~K}^{-1}$ at $295^{\circ} \mathrm{C}$. The specific heat of the source rod is $1.0653 \mathrm{~J} \mathrm{~g}^{-1} \mathrm{~K}^{-1}$ at $30{ }^{\circ} \mathrm{C}$; as the temperature increases, it increases to $1.3723 \mathrm{~J} \mathrm{~g}^{-1} \mathrm{~K}^{-1}$ at $295^{\circ} \mathrm{C}$. Lattice thermal vibration is considered to be one of the important factors affecting the specific heat of crystalline materials. The formation of oxygen vacancies will increase the lattice distortion, which is considered to cause abnormal fluctuations in the specific heat of the source rod.

The thermal conductivity $(\kappa)$ of the crystals can be calculated using the following formula:

$$
\kappa=\frac{1}{3} C_{\mathrm{v}} \lambda \bar{V}
$$

where $C_{\mathrm{v}}, \lambda$ and $\bar{V}$ denote the specific heat of the crystal, the mean free path of phonons and the average speed of phonons, respectively. Thermal conductivity versus temperature plots (4$383 \mathrm{~K}$ ) of the source rod and as-grown Yb:YAG SCFs under an inert atmosphere are shown in Fig. 10. The thermal conductivity of the Yb:YAG SCF along the $\langle 111\rangle$ direction reaches a maximum of $34.5 \mathrm{~W} \mathrm{~m}^{-1} \mathrm{~K}^{-1}$ at $40 \mathrm{~K}$. As the temperature increases, the thermal conductivity gradually approaches $7.1 \mathrm{~W}$ $\mathrm{m}^{-1} \mathrm{~K}^{-1}$ (at $100^{\circ} \mathrm{C}$ ). The thermal conductivity of the cyan source rod was significantly lower than that of the colorless crystal fiber at the same temperatures, especially in low temperature environments. The thermal conductivity at low temperatures is mainly determined by the mean free path of phonons. However, the presence of defects, impurities, and grain boundaries will greatly reduce the mean free path of phonons, which explains why the cyan source rod has lower thermal conductivity. The lower thermal conductivity will reduce the thermal management abilities of the fiber laser and increase thermal effects
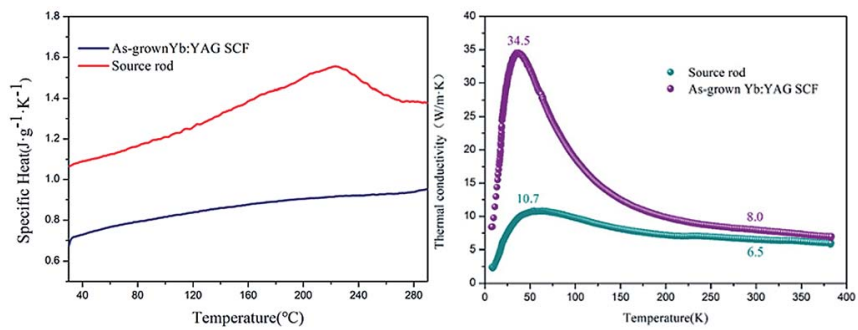

Fig. 10 Thermal properties: (left) specific heat and (right) thermal conductivity as a function of temperature. 
inside the fiber, thus affecting the laser power and beam quality.

\subsection{Formation energy of oxygen vacancies}

Calculations of the formation energy of oxygen vacancies are carried out in accordance with the following formula:

$$
\begin{aligned}
n E_{\mathrm{vac}}= & E(\text { cell with } n \text { oxygen vacancies })+\frac{n}{2} E\left(\mathrm{O}_{2}\right) \\
& -E(\text { cell without oxygen vacancies })
\end{aligned}
$$

Table 2 lists the free energy values of the system under different conditions and the oxygen vacancy formation energies. The formation energy of an oxygen vacancy is $6.51 \mathrm{eV}$ in a YAG crystal. As the $\mathrm{Yb}^{3+}$ content increases to $4 \%$ and $8 \%$, the formation energies of oxygen vacancies decrease to $6.48 \mathrm{eV}$ and $6.06 \mathrm{eV}$, respectively. We speculate that this is related to the valence state of $\mathrm{Yb}^{3+}$. When $\mathrm{Yb}^{3+}$ is converted to $\mathrm{Yb}^{2+}$, a negative electric center is formed, which induces positive electric vacancies around it. Therefore, choosing an appropriate doping concentration is also an effective way to avoid defects.

\subsection{Diameter fluctuation}

One of the most serious difficulties faced in relation to oxide single crystal fibers is controlling the fiber diameter. The situation is quite different from glass fibers; because SCFs are drawn from high temperature melt, any vibrations or irregular motion during growth can lead to diameter fluctuation.

Movement instability (fiber or source rod) and power fluctuations of the carbon dioxide laser are the main reasons for diameter fluctuations in fibers, especially for micron-sized single crystal fibers.

Through the adjustment of growth parameters and the optimization of the laser, the diameter stability of the SCFs has been obviously improved. The diameter fluctuations of two YAG SCFs are shown in Fig. 11. The average diameters of the two samples are $0.6089 \mathrm{~mm}$ and $0.8352 \mathrm{~mm}$, respectively. At the same time, the data indicates that the diameter ranges (the difference between the maximum and minimum diameters) of the two YAG SCFs are $0.02 \mathrm{~mm}$ and $0.03 \mathrm{~mm}$, representing excellent diameter control of better than $\pm 2 \%$, which is beneficial for the fabrication of cladding and related devices.

The quality of the as-grown Yb:YAG SCFs was characterized via HRXRD and Laue back-reflection measurements. The rocking curve of the (111) diffraction plane is shown in Fig. 12. The

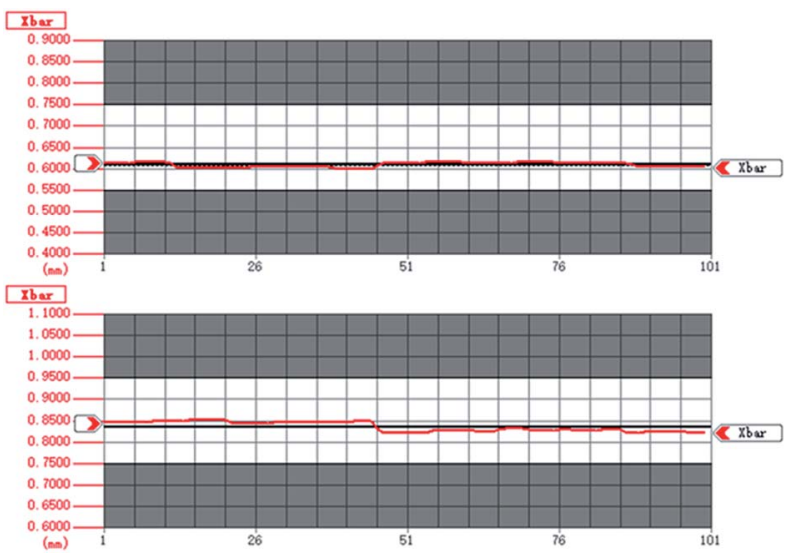

Fig. 11 The diameter fluctuations of Yb-doped YAG SCFs: (top) $\Phi 600$ $\mu \mathrm{m}$; (bottom) $\Phi 800 \mu \mathrm{m}$.

diffraction peak presents a symmetric shape without splitting. The full width at half maximum (FWHM) is estimated to be 72.2". Laue back-reflection measurements are broadly applied to investigate the crystallinity and orientation of an as-grown crystal. As can be apparently seen from Fig. 13, the characteristic Laue back-reflection patterns at different positions along the crystal fiber are uniform, clear, and bright. The above results demonstrate that the crystalline quality of the Yb:YAG SCFs is high enough, which furnishes the basis for assessing their intrinsic physical properties.

\subsection{Distribution of $\mathbf{Y b}^{3+}$}

The refractive index of the fiber can be expressed via the following equation:

$$
n(r)=\left\{\begin{array}{c}
n_{1}\left[1-2 \Delta\left(\frac{r}{a}\right)^{g}\right]^{0.5}, r \leq a \\
n_{2}, \quad r>a
\end{array}\right.
$$

where $n_{1}$ and $n_{2}$, represent the refractive indices of the core and cladding, respectively, $\Delta$ is the relative refractive index difference, $r$ and a are the radial coordinates and core radius, respectively, and $g$ stands for the distribution index of the refractive index. Due to differences in the value of $g$, the distribution of the refractive index can be divided into "gradual type" and "mutant type", as shown in Fig. 14.

In order to achieve single-mode laser output, a fiber must maintain a mutant refractive index profile, which means that

Table 2 Oxygen vacancy formation energies of Yb:YAG under different conditions

\begin{tabular}{lll}
\hline Type & $E_{\text {tot }} / \mathrm{eV}$ & $E_{\text {vac }} / \mathrm{eV}$ \\
\hline $4 \mathrm{O}_{2}$ & -33.63 \\
$\mathrm{Y}_{24} \mathrm{Al}_{40} \mathrm{O}_{96}$, without oxygen vacancies & -1303.06 \\
$\mathrm{Y}_{24} \mathrm{Al}_{40} \mathrm{O}_{88}$, with 8 oxygen vacancies in dispersed states & -1217.32 & -1298.68 \\
$\mathrm{Y}_{23} \mathrm{Yb}_{1} \mathrm{Al}_{40} \mathrm{O}_{96}$, without oxygen vacancies & -1213.20 \\
$\mathrm{Y}_{23} \mathrm{Yb}_{1} \mathrm{Al}_{40} \mathrm{O}_{88}$, with 8 oxygen vacancies & -1290.45 \\
$\mathrm{Y}_{22} \mathrm{Yb}_{2} \mathrm{Al}_{40} \mathrm{O}_{96}$, without oxygen vacancies & -1208.27 & 6.51 \\
$\mathrm{Y}_{22} \mathrm{Yb}_{2} \mathrm{Al}_{40} \mathrm{O}_{88}$, with 8 oxygen vacancies & & 6.48
\end{tabular}




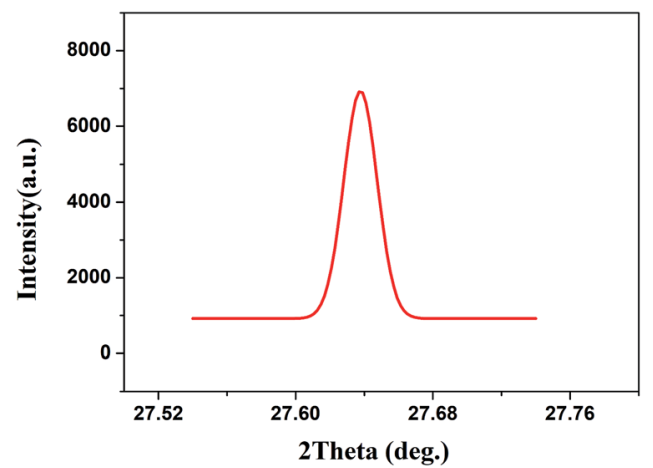

Fig. 12 X-ray rocking curve of the (111) diffraction plane of the Yb:YAG SCFs.

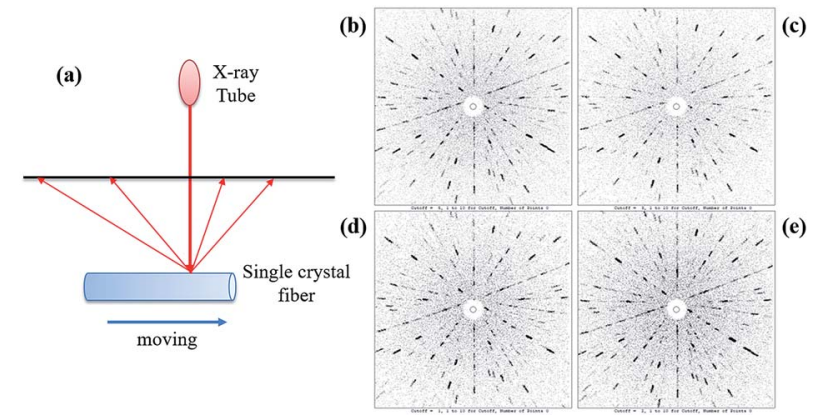

Fig. 13 (a) A schematic diagram showing the positions of the Laue back-reflection measurements on the crystal fiber. (b-e) Characteristic Laue back-reflection patterns at different positions with the $\mathrm{X}$-ray beam hitting the crystal fiber.

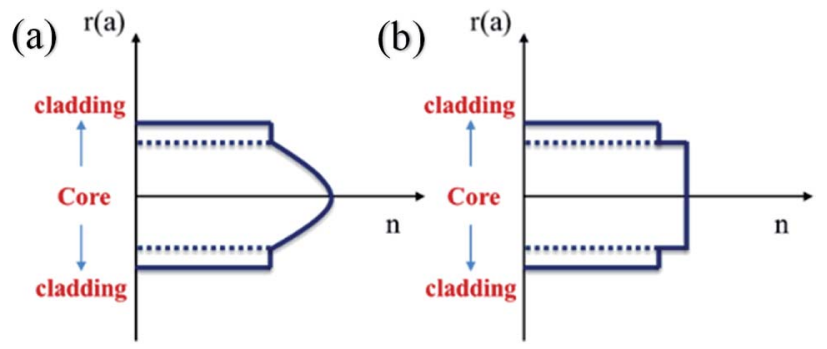

Fig. 14 The refractive index of a SCF: (a) gradual type and (b) mutant type.

the refractive index of the core must be uniform. For doped crystals, it is necessary to ensure the uniformity of rare earth ion distribution. We determined the elemental compositions of the

Table 3 Elemental compositions of the source rod and an as-grown $\mathrm{SCF}$

\begin{tabular}{lllll}
\hline & $\mathrm{Al}(\mathrm{mass} \%)$ & $\mathrm{Y}(\mathrm{mass} \%)$ & $\mathrm{Yb}(\operatorname{mass} \%)$ & $\mathrm{Yb} / \mathrm{Y}(\mathrm{at} \%)$ \\
\hline Source rod & 21.2 & 75.3 & 3.46 & 2.36 \\
As-grown SCF & 25.5 & 72.1 & 3.31 & 2.35
\end{tabular}

source rod and crystal fibers via X-ray fluorescence (XRF). As shown in Table 3, the concentrations of $\mathrm{Yb}^{3+}$ in the source rod and the as-grown SCF were measured to be 2.36 and 2.35, respectively. The calculated segregation coefficient of $\mathrm{Yb}^{3+}$ is close to 1.

The concentrations of $\mathrm{Yb}^{3+}$ along the radial and axial directions are shown in Fig. 15. We found that $\mathrm{Yb}^{3+}$ is uniformly distributed in both the radial and axial directions without significant ion-rich regions, which is a key factor for achieving single-mode lasers.

\subsection{Propagation loss}

The following standard formulas were used to calculate the attenuation coefficients on the log scale:

$$
\alpha_{\mathrm{dB}}=-\frac{1}{L} 10 \log \frac{P_{\text {out }}}{P_{\text {in }}}
$$

where $L, P_{\text {out }}$, and $P_{\text {in }}$ denote the fiber length, the output laser power, and the input laser power, respectively. Fresnel reflection loss is included in the total fiber loss. Therefore, both ends of the fiber are polished to laser quality and the test results were also corrected for Fresnel reflections. We selected two parts of different lengths from the same fiber; the Fresnel reflection losses and the attenuation coefficients of the two samples are considered to be equal at the same power, thus eliminating any error caused by Fresnel reflections. The obtained measurement results are presented in Table 4 . We found that the propagation attenuation coefficient is around $0.01 \mathrm{~dB} \mathrm{~cm}^{-1}$, indicating that the grown crystal fibers are of good quality and possess excellent optical waveguiding performance.

\subsection{Lasing with a Yb:YAG SCF}

The laser wavelength is centered at $1030.82 \mathrm{~nm}$, which is consistent with the emission spectrum (shown in Fig. 16). Fig. 17 shows the output laser power as a function of the absorbed pump power under CW pumping. A maximum output power of $3.62 \mathrm{~W}$ was measured for an output mirror with transmittance of $30 \%$ at an absorbed pump power of $16.75 \mathrm{~W}$. Taking into account all the Fresnel reflection losses and the un-

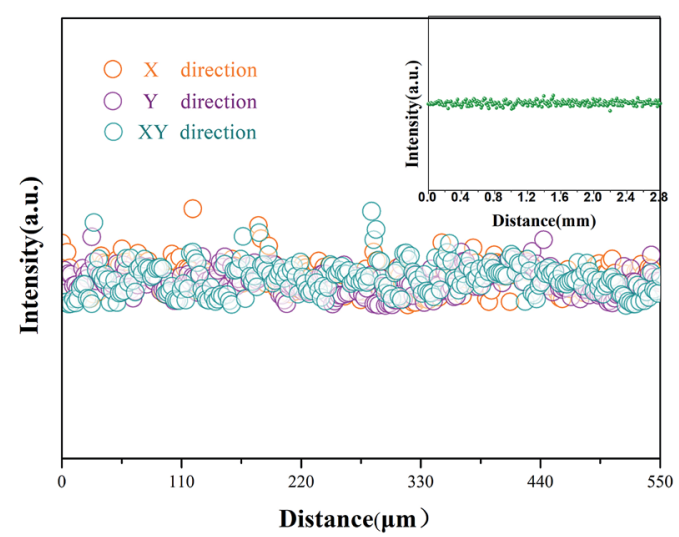

Fig. 15 The radial distribution of $\mathrm{Yb}^{3+}$. The inset shows the distribution of $\mathrm{Yb}^{3+}$ along the axial direction. 
Table 4 Attenuation coefficients at different powers

\begin{tabular}{|c|c|c|c|c|c|c|c|}
\hline Sample & $L(\mathrm{~cm})$ & $P_{\text {in }}(\mathrm{W})$ & $P_{\text {out }}(\mathrm{W})$ & $\begin{array}{l}P_{\text {out }} / P_{\text {in }} \text { (without } \\
\text { subtraction of the reflection loss) }\end{array}$ & $\begin{array}{l}P_{\text {out }} / P_{\text {in }} \text { (with } \\
\text { subtraction of the reflection loss) }\end{array}$ & $\begin{array}{l}\text { Reflection } \\
\text { loss }\end{array}$ & $\alpha_{\mathrm{dB}}$ \\
\hline Fiber 1 & 0.8 & 1.034 & 0.914 & $88.39 \%$ & $99.76 \%$ & $11.37 \%$ & $0.013 \mathrm{~dB} \mathrm{~cm}^{-1}$ \\
\hline Fiber 1 & 0.8 & 2.003 & 1.763 & $88.02 \%$ & $99.72 \%$ & $11.7 \%$ & $0.015 \mathrm{~dB} \mathrm{~cm}^{-1}$ \\
\hline Fiber 2 & 1.8 & 2.003 & 1.756 & $87.67 \%$ & $99.37 \%$ & $11.7 \%$ & $0.015 \mathrm{~dB} \mathrm{~cm}^{-1}$ \\
\hline Fiber 1 & 0.8 & 3.016 & 2.637 & $87.43 \%$ & $99.84 \%$ & $12.41 \%$ & $0.008 \mathrm{~dB} \mathrm{~cm}^{-1}$ \\
\hline Fiber 2 & 1.8 & 4.005 & 3.479 & $86.87 \%$ & $99.51 \%$ & $12.64 \%$ & $0.012 \mathrm{~dB} \mathrm{~cm}^{-1}$ \\
\hline
\end{tabular}

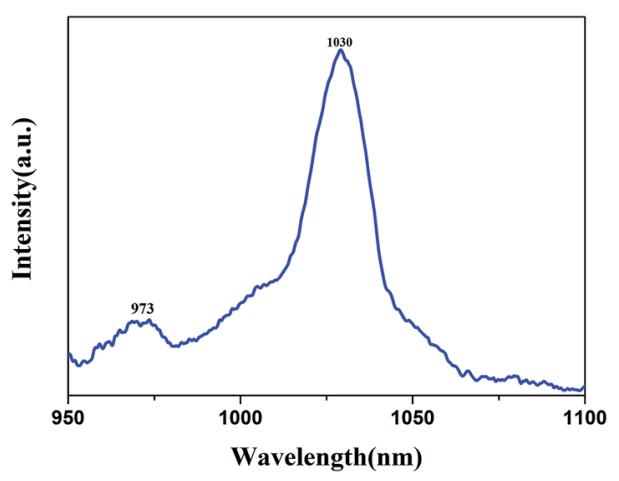

Fig. 16 The emission spectrum of a Yb:YAG SCF.

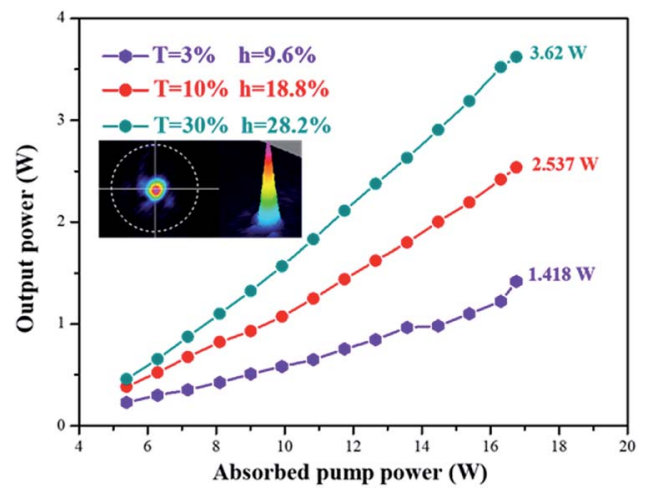

Fig. 17 The output laser power as a function of absorbed pump power with different output mirrors.

absorbed pump power, the slope efficiency was calculated to be $28.2 \%$ with a lasing threshold of $3.92 \mathrm{~W}$. The output beam satisfies a Gaussian distribution and the beam quality factor $M^{2}$ was measured to be 1.45 , which could be further optimized by using a more compact resonator.

\section{Conclusions}

In this paper, colorless Yb:YAG SCFs with a length-to-diameter ratio exceeding 320:1 were successfully grown in both aerobic and anaerobic environments via a LHPG method. The diameter fluctuation is less than $2 \%$, indicating that the crystal growth process is stable and the crystal fibers are relatively uniform. By means of single crystal X-ray diffraction, XPS and Raman spectroscopy, we found that the colorless Yb:YAG SCFs possess larger unit cell parameters, fewer oxygen vacancies and more obvious $\mathrm{AlO}_{4}$ and $\mathrm{Yb}-\mathrm{O}$ vibrations than the cyan Yb:YAG crystal source rod. Therefore, we conclude that the discoloration of Yb:YAG crystals is caused by oxygen vacancies, and the formation energy of oxygen vacancies decreases as the $\mathrm{Yb}^{3+}$ content increases. We believe that the large specific surface area of the grown Yb:YAG SCFs, the low $\mathrm{Yb}^{3+}$ doping concentration and the surface tension convection of the LHPG method are the main factors in avoiding defects.

At the same time, some optical and thermal properties of the cyan Yb:YAG crystal source rod and colorless Yb:YAG SCFs were measured. The discolored Yb:YAG crystal, with discoloration caused by oxygen vacancies, has lower infrared transmittance and thermal conductivity, which will reduce the laser output and the beam quality dramatically. Meanwhile, the as-grown SCFs were confirmed to be of high quality from rocking curve and Laue back-reflection measurements, and the propagation attenuation coefficient is around $0.01 \mathrm{~dB} \mathrm{~cm}^{-1}$. Through EPMA measurements, we found that $\mathrm{Yb}^{3+}$ is uniformly distributed in the YAG SCFs, which is necessary for achieving single-mode lasers. Based on this, we carried out laser experiments under different conditions and achieved a maximum continuous-wave (CW) output power of $3.62 \mathrm{~W}$ with a slope efficiency of $28.2 \%$. In conclusion, all properties, including the small diameter fluctuation, good crystal quality, good thermal conductivity and uniform ion distribution, prove that the colorless Yb:YAG SCFs are a promising host material for single-mode lasers.

\section{Conflicts of interest}

There are no conflicts to declare.

\section{Acknowledgements}

We gratefully acknowledge financial support from the National key Research and Development Program of China (Grant No. 2016YFB1102201, 2018YFB0406502), the Young Scholars Program of Shandong University (Grant No. 2015WLJH36), and the 111 Project 2.0 (Grant No: BP2018013). 


\section{Notes and references}

1 J. Nilsson and D. N. Payne, Science, 2011, 332(6032), 921-922. 2 D. J. Richardson, J. Nilsson and W. A. Clarkson, J. Opt. Soc. Am. B, 2010, 27(11), B63-B92.

3 U. Keller, Nature, 2003, 424, 831.

4 P. Norvig, D. A. Relman, D. B. Goldstein, et al., Nature, 2010, 463(7), 26.

5 Q. Hu, Z. T. Jia, et al., CrystEngComm, 2017, 19, 537-545.

6 C. Kränkel, D. T. Marzahl, et al., Laser Photonics Rev., 2016, 10(4), 548.

7 J. Shu, Z. T. Jia, et al., CrystEngComm, 2018, 20, 6741.

8 S. I. Parker, Nucl. Instrum. Methods Phys. Res., Sect. A, 1997, 395(3), 328-343.

9 N. Soleimani, B. Ponting, et al., Proc. SPIE, 2014, 8959, 895903.

10 J. A. Harrington, Proc. SPIE, 2014, 8959, 895902.

11 X. Délen and A. Aubourg, Proc. SPIE, 2015, 9342, 934202934208.

12 T. A. Parthasarathy, Opt. Eng., 2010, 49(9), 094302.

13 J. W. Dawson, Opt. Express, 2008, 16(17), 13240-13266.

14 T. J. Bridges, J. S. Hasiak and A. R. Strnad, Opt. Lett., 1980, 5(3), 85-86.

15 W. Que, Y. Zhou, Y. L. Lam, Y. C. Chan and C. H. Kam, J. Mod. Opt., 2000, 47(6), 1127-1136.

16 R. M. Ribeiro, A. B. A. Fiasca, P. A. M. dos Santos, M. R. B. Andreeta and A. C. Hernandes, Opt. Mater., 1998, 10(3), 201-205.

17 R. K. Nubling and J. A. Harrington, Appl. Opt., 1997, 36(24), 5934-5940.

18 S. Bera, C. D. Nie, et al., Opt. Mater., 2018, 75, 44-48.

19 P. Z. Yang, P. Z. Deng, J. Xu and Z. W. Yin, J. Cryst. Growth, 2000, 216(1-4), 348-351.

20 Y. L. Yang, L. H. Ye, R. J. Bao, S. M. Li, P. X. Zhang, M. Xu and Y. Hang, Infrared Phys. Technol., 2018, 91, 85-89.
21 T. A. Parthasarathy, R. S. Hay, G. Fair and F. K. Hopkins, Opt. Eng., 2010, 49, 094302.

22 W. Kim, C. Florea, C. Baker, D. Gibson and L. B. Shaw, Proc. SPIE, 2012, 8547, 85470K.

23 C. D. Nie, S. Bera and J. A. Harrington, Opt. Express, 2016, 24, 15522-15527.

24 D. P. Bour, D. B. Gilbert and K. B. Fabian, IEEE Photonics Technol. Lett., 1990, 2, 173-174.

25 X. D. Mu, S. Meissner and H. Meissner, Proc. SPIE, 2015, 9342, 934209.

26 L. E. Johnson, J. E. Geusic and L. G. Van Uiten, Appl. Phys. Lett., 1965, 7, 127-130.

27 P. Lacovara, H. K. Choi, C. A. Wang, et al., Opt. Lett., 1991, 16(14), 1089-1091.

28 X. Délen, Y. Zaouter, et al., Opt. Lett., 2013, 38(2), 109-111. 29 A. Yoshikawa, G. Boulon, et al., J. Appl. Phys., 2003, 94, 5479.

30 V. I. Chani, A. Yoshikawa, et al., Mater. Res. Bull., 2000, 35(10), 1615-1624.

31 X. Délen, S. Piehler, J. Didierjean, et al., Opt. Lett., 2012, 37(14), 2898.

32 P. Yang, P. Deng and Z. Yin, J. Lumin., 2002, 97(1), 51-54.

33 H. Yin, P. Deng and F. Gan, J. Appl. Phys., 1998, 83(7), 3825.

34 G. Kresse and J. Furthmüller, Comput. Mater. Sci., 1996, 6(1), 15.

35 J. P. Perdew, K. Burke and M. Ernzerhof, Phys. Rev. Lett., 1996, 77(18), 3865.

36 G. Kresse and D. Joubert, Phys. Rev. B: Condens. Matter Mater. Phys., 1999, 59(3), 1758.

37 G. L. Kabongo, G. H. Mhlongo, B. M. Mothudi, K. T. Hilliebd, H. C. Swart and M. S. Dhlamini, Mater. Lett., 2014, 119(15), 71-74.

38 Y. F. Chen, P. K. Lim, S. J. Lim and Y. J. Yang, J. Raman Spectrosc., 2003, 34(11), 882-885. 